\title{
DISTONIA DE TORSĀO E SÍNDROME PARKINSONIANA
}

\author{
José Geraldo Camargo lima * \\ Pedro Camilo A. Pimentel ** \\ ANTONio LuChesi Filho *** \\ BONIFACIO MENEZES *** \\ WALDEMAR BITTENCOURT ***
}

A distonia de torsão teve o seu quadro clínico bem definido e individualizado dentro do grupo das hipercinesias involuntárias por Ziehen em 1910 (cit. por Austregésilo Filho ${ }^{1}$ ) e a sua base orgânica enfatizada por Babinski em 1900 (cit. por Costa $^{3}$ ) e por Oppenheim em 1911 (cit. por Austregésilo Filho ${ }^{1}$ ). De sinonimia variada - distonia lordótica progressiva, distonia musculorum deformans, espasmo de torsão e enfermidade de Ziehen-Oppenheim - era considerada uma entidade extremamente rara, tornando-se mais freqüente após as epidemias de encefalite letárgica. Predominando no sexo masculino, iniciando-se nas idades as mais variáveis, apresenta, em pequeno número de casos, um fator hereditário de importância, e tem a encefalite letárgica como a etiologia mais freqüente. Para autores como Brain ${ }^{2}$, Denny-Brown ${ }^{4}$ e Ford ${ }^{5}$ o torcicolo espasmódico, distonia sediada na musculatura cervical, nada mais é do que uma forma localizada desse complexo grupo nosológico.

A possibilidade de associação entre uma síndrome distônica e o parkinsonismo é referida na literatura $1,6,7,8,9,10,11$, sendo a raridade desse fato sempre realçada.

\section{MATERIAL, MÉTODO E RESULTADOS}

o material consta de 4 pacientes com síndrome pårkinsoniana associada a uma sindrome distônica. Essa cifra, no cômputo geral de pacientes parkinsonianos estudados (257), mostra uma freqüência de 1,5\%. A síndrome distônica em todos os pacientes se restringia à região cervical, manifestando-se como torcicolo espasmódico. Outras características clínicas desses pacientes estão assinaladas na tabela 1.

\section{COMENTARTOS}

De 257 pacientes com síndrome parkinsoniana, 4 (1,5\%) apresentaram associadamente distonia de torsão. Em todos essa distonia se manifestou como torcicolo espasmódico. Contrariamente à citada predominância dessa

Trabalho do Departamento de Neurologia e Neurocirurgia da Escola Paulista de Medicina, apresentado no $\mathrm{V}$ Congresso Brasileiro de Neurologia e III Congresso Brasileiro de Eletrencefalografia e Neurofisiologia Clínica (São Paulo 12-15 julho 1972): * Professor Adjunto; ** Assistente; *** Médicos estagiários. 
síndrome distônica no sexo masculino, todos os nossos pacientes eram do sexo feminino. A idade de início da síndrome parkinsoniana e da distonia - 12 a 40 anos e 18 a 40 anos, respectivamente - ao lado de outros elementos anamésticos característicos permitiram em todos os casos, admitir a etiologia encefalítica, o que está concorde com a maioria dos autores, para os quais outras causas como intoxicação, distúrbios vasculares e degeneração primária dos núcleos da base são excepcionais.

\begin{tabular}{|c|c|c|c|c|}
\hline Casos & 1 & 2 & 3 & 4 \\
\hline Nome & N.B.O. & L.C. & I.B.S. & I.A. \\
\hline Sexo & $\mathbf{F}$ & F & $\mathbf{F}$ & $\mathbf{F}$ \\
\hline Duração da doença & 18 anos & 4 anos & 1 ano & 5 anos \\
\hline Idade de inicio do Parkinson & 12 & 28 & 40 & 40 \\
\hline Topografia do Parkinson & unil. $\mathbf{E}$ & unil. E. & $\begin{array}{l}\text { bilat. } \\
\text { pred. D. }\end{array}$ & unil. $\mathbf{E}$. \\
\hline Sinal predominante do Parkinson & 一 & hipert. & hipert. & hipert. \\
\hline Idade de início da distonia torsão & 18 & 26 & 40 & 40 \\
\hline
\end{tabular}

Tabela 1 - Aspectos clinicos dos 4 pacientes com distonia de torsão associada a sindrome parkinsoniana.

Como na maioria dos casos de distonia de torsão pura, também em nossos pacientes não houve qualquer componente familiar de importância. Em um paciente (caso 2) a distonia precedeu a síndrome parkinsoniana, nos outros foi concomitante ou sucedeu por 6 anos (caso 1). A síndrome parkinsoniana associada era predominantemente unilateral hipertônica; em um paciente (caso 2) havia outro sinal neurológico associado (deficit de convergência).

\section{R E S U M O}

Os autores relatam 4 casos de distonia de torsão, do tipo torcicolo espasmódico, associada a síndrome parkinsoniana. Todos os pacientes eram do sexo feminino e as idades de início da sindrome parkinsoniana e da síndrome distônica variaram de 12 a 40 anos e de 13 a 40 anos, respectivamente. Em apenas uma paciente a distonia precedeu ao parkinsonismo. A síndrome parkinsoniana era predominantemente unilateral e hipertônica. A etiologia encefalítica foi a aventada em todos os casos. 


\section{Torsion dystonia and Parkinson's syndrome}

Four cases of torsion dystonia of the spasmodic torticollis type associated to Parkinson's syndrome are reported. All the patients were female and the ages at the begining of the parkinsonism and the dystonic syndrome varied respectively from 12 to 40 and from 18 to 40 years. In just one patient the dystonia preceded the parkinsonism. The Parkinson's syndrome was predominantly unilateral and hypertonic. The encephalitic etiology was suggested in all the cases.

\section{REFERENCIAS}

1. AUSTREgésilo FIlHo, A. - Distonia de Torsão. Ed. Irmãos Di Giorgio, Rio de Janeiro, 1945.

2. BRAIN, R. - Diseases of the Nervous System. Sixth Edition. Oxford University Press, London, 1962.

3. CostA, D. G. - Torcicolo espasmódico. A propósito de um caso operado. Arq. Neuro-Psiquiat. (São Paulo) 2:392, 1944.

4. DENNY-BROWN, D. - The Basal Ganglia and their Relation to Disorders of Movement. Oxford University Press. Amen House, London, 1962.

5. FORD, F. R. - Diseases of the Nervous System in Infancy, Childhood and Adolescence. Third Edition. Charles C. Thomas, Springfield (Illinois), 1952.

6. KÄFER, J. P. \& POCH, G. P. - Neurologia. Ed. Lopes Libreros, Buenos Aires, 1969.

7. STERLING, M. W. \& ROSENBLUM, S. - Syndrome de spasme de torsion avec une syncinésie spasmodique particulière dans le parkinsonisme encephalitique. Rev. neurol. (Paris) 2:486, 1928.

8. STERLING, M. W. \& STEIN, W. - Hemiballisme, dystonie et tremblement parkinsonien au cours d'une sclerose en plaques. Rev. neurol. (Paris) 65:84T, 1936.

9. STUTTE, H. - On striatal torticolis with special reference to its connections with extrapiramidal disturbances. Resumo in Excerpta Med. Sect. VIII Neurology and Psychiat. 1:369, 1948.

10. ZEMAN, W. - Pathology of the torsion dystonias (dystonias musculorum deformans). Neurology (Minneapolis) 20:79, 1970.

11. ZEMAN, W. \& DYKEN, P. - Dystonia musculorum deformans: clinical, genetic and pathoanatomical studies. Psychiat. Neurol. Neurochir. (Amsterdam) 70:77, 1967.

Departamento de Neurologia e Neurocirurgia - Escola Paulista de Medicina Caixa Postal 5496 - 01000 São Paulo, SP - Brasil. 